\title{
A Four-Step Global Reaction Mechanism for CFD Simulations of Flexi-Fuel Burner for Gas Turbines
}

\author{
A. Abou-Taouk ${ }^{1}$, I. R. Sigfrid ${ }^{2}$, R. Whiddon ${ }^{3}$ and L. E. Eriksson ${ }^{4}$ \\ ${ }^{1,4}$ Department of Applied Mechanics, Chalmers University of Technology, \\ Hörsalsvägen 7A, 41296 Gothenburg, Sweden, abdallah@chalmers.se, lee@chalmers.se \\ ${ }^{2}$ Department of Energy Sciences, Lund University, \\ Ole Römers väg 1, 22100 Lund, Sweden, Ivan.Sigfrid@energy.Ith.se \\ ${ }^{3}$ Department of Combustion Physics, Lund University \\ Box 118, 22100 Lund, Sweden, ronald.whiddon@forbrf.lth.se
}

\begin{abstract}
A reduced four-step scheme for Computational Fluid Dynamics (CFD) analysis is presented here in the context of industrial type laboratory combustor. The present scheme has been optimized for a syngas mixture consisting of $10 \% \mathrm{CH}_{4}, 22.5 \% \mathrm{CO}$ and $67.5 \% \mathrm{H}_{2}$ by volume, and for a methane-air mixture. The optimization of the global scheme is done by comparing with the detailed San Diego mechanism using perfectly stirred reactor (PSR) and laminar flame speed calculations. The four-step global scheme has been applied to the CFD analysis of a swirl-stabilized flexi-fuel burner. Both reacting and non-reacting cases has been computed, using a hybrid Unsteady RANS/Large Eddy Simulation (URANS/LES) technique. Comparisons between CFD results and experimental data in the form of Particle Image Velocimetry (PIV) data, Planar Laser Induced Fluorescence (pLIF) and Proper Orthogonal Decomposition (POD) analysis from an atmospheric burner test rig at Lund University are presented. The CFD results scheme show good agreement with the experimental data.
\end{abstract}

\section{Introduction}

Combustion of fossil fuels has provided the majority of our energy needs in the past and will remain the dominating energy conversion process for at least the next 50 years. Therefore combustion remains a key energy technology for the foreseeable future where improved combustion technology in terms of efficiency and pollutant emissions is important. Regulations to reduce emissions from fossil fuels have led to development of combustor technology towards fuel-flexibility and increased use of bio fuels. Prediction of combustor performance, including efficiency, ignition, flame stability and emissions characteristics, requires both detailed modeling and advanced measuring techniques. Building on previous work by Abou-Taouk et al. (2011), the present work involves syngas fuel consisting of $10 \%$ $\mathrm{CH}_{4}, 22.5 \% \mathrm{CO}$ and $67.5 \% \mathrm{H}_{2}$ by volume. For this type of complex fuel, a detailed reaction mechanism requires a large computational time. To limit the computational time the number of reactants and species has to be limited to a few global reactions. Several different reduced reaction mechanisms of syngas-mixtures exist in the literature [1-6]. Most of them do not include the $\mathrm{CH}_{4}$ species.

The optimization of the present reduced global scheme is based on a strategy where detailed chemistry in the form of both PSR calculations and laminar flame speed calculations in the CHEMKIN software are applied. The method chosen is based on the idea that the global mechanism may be optimized for an equivalence ratio range $\varphi=0.4-1.8$ by the addition of a correction function for $\varphi=0.8-1.8$. The correction function is introduced into the $\mathrm{CO}-\mathrm{CO}_{2}$ elementary reaction and is necessary to match the detailed and the global 
reaction mechanism at higher $(\varphi)$. A premixed down-scaled $4^{\text {th }}$-generation Dry Low Emission (DLE) burner supplied by Siemens Industrial Turbomachinery (SIT), is used in this work, and called hereafter the SIT flexi-fuel burner. It was developed to be fuel flexible. This combustor is comprised by a lean premixed Main part, a partially premixed Pilot and a confined Rich Pilot Lean (RPL) flame which produces radicals and heat that support both the pilot and the main flame.

\section{Experimental Setup}

For validation purposes a set of experiments have been performed on the SIT flexi-fuel burner. The burner has also been investigated in previous publications [7-10].

\subsection{System description}

The burner (Figure 1), which has three concentric sectors, is connected to a square liner. The liner is built in two parts. The first part is a $260 \mathrm{~mm}$ quartz liner in order to gain line of sight access for laser diagnostics. The second part is a $400 \mathrm{~mm}$ stainless steel liner in order to avoid influence from the contraction on the flow field. The liner has a $105 \mathrm{~cm}^{2}$ cross section. After the liner a cylindrical contraction lead the combusted gases to a ventilated exhaust. The cross section at the end cylinder is $53 \mathrm{~cm}^{2}$.

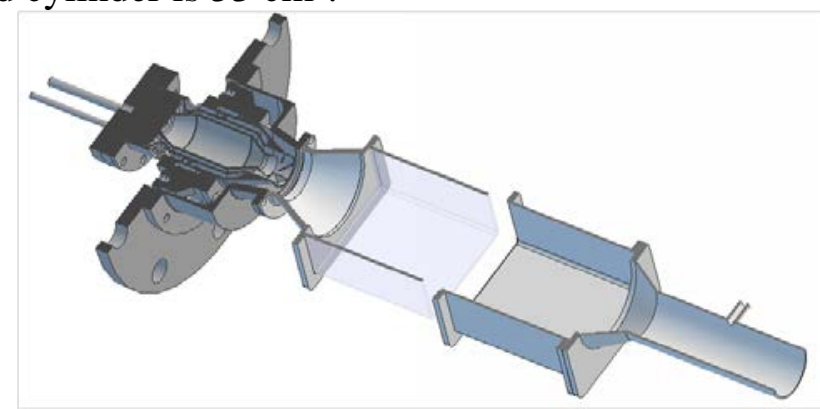

Figure 1: The three sector burner with line of sight access for laser diagnostics

All three burner sectors have separate fuel supply, and the central sector (RPL) has its own air supply. These flows are controlled by Alicat Scientific Mass Flow Controllers. The air to the 2 other sections, the Pilot and the Main, is supplied from two Rieschle SAP 300 blowers. The air split between the Pilot and the Main are set by the pressure drop over the corresponding sector resulting in $21 \%$ of the air going to the Pilot section and $79 \%$ to the Main sector [9]. All of the air supplied to the burner is preheated to $650 \mathrm{~K}$ before entering the combustion zone. The total air and fuel flow through the burner was $55 \mathrm{~g} / \mathrm{s}$, of which $1.3 \mathrm{~g} / \mathrm{s}$ goes to the RPL. The $\varphi$ in the RPL was 0.64. In the Pilot and the Main the equivalence ratio was set to 0.31 . The three systems together give an adiabatic flame temperature corresponding to $1490 \mathrm{~K}$.

\subsection{Measurement setup}

Two laser based measurements techniques are individually performed. The first is with a high speed PIV system with a diode pumped Nd:YLF laser, Dantec Dynamics DualPower TR. The camera used is a Dantec Dynamics Speed Sense 9060. The parameters used for PIV is stated in Figure 2. The second laser based technique implemented is pLIF. Fluorescence from the $\mathrm{OH}$ molecule, a combustion intermediate, is excited using an $\mathrm{Nd}$ :YAG pumped dye laser. The dye used is rhodamine 6G in ethanol which, with doubling, is equal to the OH Q1(8) 
transition; the laser energy used is approximately $4 \mathrm{mj} / \mathrm{pulse}$. The laser beam is formed into an expanding sheet using a $-12 \mathrm{~mm}$ cylindrical lens and a $500 \mathrm{~mm}$ spherical lens. The beam height is approximately $107 \mathrm{~mm}$ at the quartz liner entry face and $120 \mathrm{~mm}$ at the exit face, with the beam waist focused at the burner major flow axis.

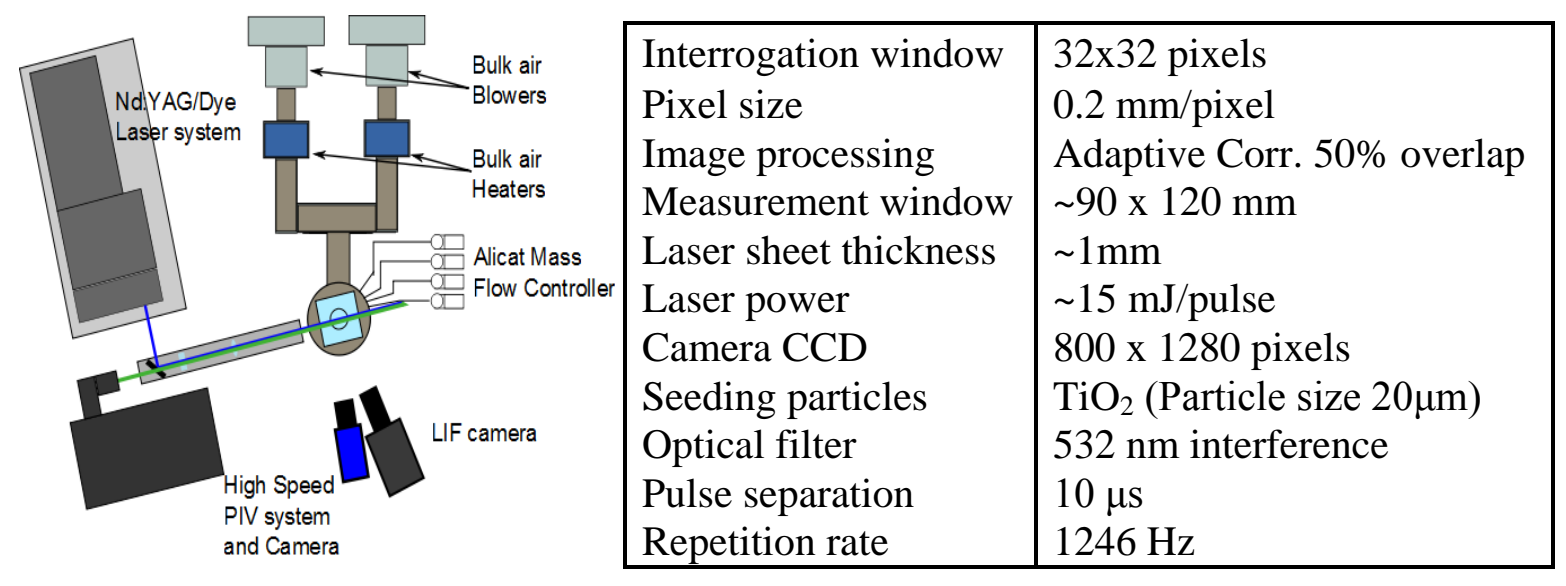

Figure 2: The measurement setup including PIV and LIF lasers and settings

\section{Kinetic Optimization}

Simplified chemistry is usually required in CFD simulations together with combustion since the simulation often are limited by the run-time and convergence requirements. Global reaction mechanisms are well suited for CFD calculation since they are based on Arrhenius rates and can straightforwardly be implemented into CFD-codes. The four-step global reaction mechanism for syngas-air mixture is optimized against a detailed reference mechanism [11,12] for perfectly stirred reactor (PSR) calculations and laminar flame speeds.

\subsection{Global reaction mechanism}

The present optimized global scheme consists of four reactions corresponding to the methane oxidation into $\mathrm{CO}$ and $\mathrm{H}_{2}$, the equilibrium water gas shift reaction $\left(\mathrm{CO}+\mathrm{H}_{2} \mathrm{O}=\mathrm{CO}_{2}\right.$ $+\mathrm{H}_{2}$ ), the $\mathrm{CO}-\mathrm{CO}_{2}$ and the $\mathrm{H}_{2}-\mathrm{H}_{2} \mathrm{O}$ equilibrium reactions.

Table 1: Pre-exponential factor $A$, activation energy $E_{a}$, and temperature exponent $\beta$

\begin{tabular}{|l|c|c|c|}
\hline \multicolumn{1}{|c|}{ Reaction } & $\mathrm{A}$ & $\mathrm{E}_{\mathrm{a}}$ [cal/mole] & $\beta$ \\
\hline $\mathrm{CH}_{4}+0.5 \mathrm{O}_{2} \rightarrow \mathrm{CO}+2 \mathrm{H}_{2}$ & $9.660840 \mathrm{e} 12$ & 35000 & 0 \\
$\mathrm{H}_{2}+0.5 \mathrm{O}_{2} \leftrightarrow \mathrm{H}_{2} \mathrm{O}$ & $1.428428 \mathrm{e} 18$ & 40445 & -1.1682 \\
$\mathrm{CO}+0.5 \mathrm{O}_{2} \leftrightarrow \mathrm{CO}_{2}$ & $5.531351 \mathrm{e} 14$ & 40735 & 0 \\
$\mathrm{CO}+\mathrm{H}_{2} \mathrm{O} \leftrightarrow \mathrm{CO}_{2}+\mathrm{H}_{2}$ & $1.791791 \mathrm{e} 12$ & 20845 & 0 \\
\hline
\end{tabular}

The reaction rates [mole, $\mathrm{cm}^{3}, \mathrm{~K}, \mathrm{~s}$ ] for the four reactions are visible below. The reaction orders for $\mathrm{RR}_{1}$ and $\mathrm{RR}_{2}$ are also included in the optimized loop. In the four-step global reaction mechanism $A$ is the pre-exponential factor, $E_{a}$ is the activation energy, $R$ is the universal gas constant, $\mathrm{T}$ is the temperature and $\beta$ is the temperature exponent. Figure 3 shows the correction function included in the reaction rate $R_{3}$ and equation 1 is used to fit this curve. The function $\mathrm{f}$ is originally coming from Franzelli et al. [6] but here we use new constants visible in table 2 . No correction is needed for $\varphi<0.8$, since the optimized four-step 
global reaction mechanism predicts temperatures, laminar flame speeds and emissions sufficiently well in this region.

$$
\begin{aligned}
& \mathrm{RR}_{1}=\mathrm{A}_{1} \mathrm{~T}^{\beta_{1}} \mathrm{e}^{\frac{-\mathrm{E}_{\mathrm{a} 1}}{\mathrm{RT}}}\left[\mathrm{CH}_{4}\right]^{0.37}\left[\mathrm{O}_{2}\right]^{1.3991} \\
& \mathrm{RR}_{2}=\mathrm{A}_{2} \mathrm{~T}^{\beta_{2}} \mathrm{e}^{\frac{-\mathrm{E}_{\mathrm{a} 2}}{\mathrm{RT}}}\left[\mathrm{H}_{2}\right]^{0.5}\left[\mathrm{O}_{2}\right]^{1.1} \\
& \mathrm{RR}_{3}=\mathrm{f}(\varnothing) \mathrm{A}_{3} \mathrm{~T}^{\beta_{3}} \mathrm{e}^{\frac{-\mathrm{E}_{\mathrm{a} 3}}{\mathrm{RT}}}[\mathrm{CO}]^{1}\left[\mathrm{O}_{2}\right]^{0.5} \\
& \mathrm{RR}_{4}=\mathrm{A}_{4} \mathrm{~T}^{\beta_{4}} \mathrm{e}^{\frac{-\mathrm{E}_{\mathrm{a} 4}}{\mathrm{RT}}}[\mathrm{CO}]^{1}\left[\mathrm{H}_{2} \mathrm{O}\right]^{1}
\end{aligned}
$$

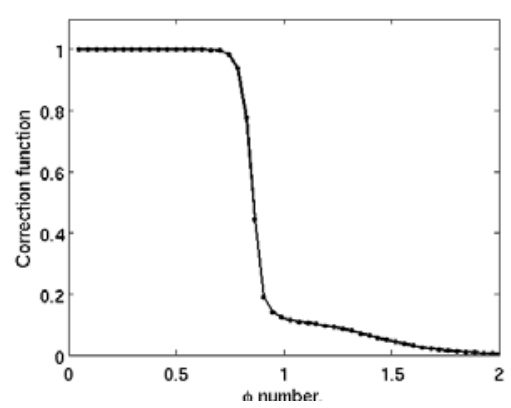

Figure 3: The correction function $\mathrm{f}$

In the region $0.8<\varphi<1.8$ the production of $\mathrm{CO}_{2}$ is reduced for rich mixtures when the $\varphi$ increases to match the laminar flame speed.

$$
f(\varnothing)=\frac{1}{2}\left[1+\tanh \left(\frac{\emptyset_{0,2}-\theta}{\sigma_{0,2}}\right)\right]+\frac{B}{2}\left[1+\tanh \left(\frac{\theta-\emptyset_{1,2}}{\sigma_{1,2}}\right)\right]+\frac{c}{2}\left[1+\tanh \left(\frac{\theta-\emptyset_{2,2}}{\sigma_{2,2}}\right)\right]\left[1+\tanh \left(\frac{\emptyset_{3,2}-\theta}{\sigma_{3,2}}\right)\right]
$$

Table 2: Constants in correction function $\mathrm{f}$

\begin{tabular}{|c|c|c|c|c|c|c|c|c|c|c|}
\hline & $\emptyset_{0,2}$ & $\sigma_{0,2}$ & $\mathrm{~B}$ & $\emptyset_{1,2}$ & $\sigma_{1,2}$ & $\mathrm{C}$ & $\emptyset_{2,2}$ & $\sigma_{2,2}$ & $\emptyset_{3,2}$ & $\sigma_{3,2}$ \\
\hline $\mathrm{f}$ & 0.86 & 0.055 & 0.02 & 1 & 0.14 & 0.063 & 0.92 & 0.03 & 1.4 & 0.35 \\
\hline
\end{tabular}

\subsection{Results of the laminar flame speed simulations}

Figure 4 shows laminar flame speeds and adiabatic flame temperatures for syngas and methane-air mixtures using the detailed mechanisms (Gri Mech 3.0 and San Diego) and the present four-step scheme. The inlet temperature is set to $650 \mathrm{~K}$ and atmospheric pressure is used. The agreement is excellent for the syngas mixture while the agreement for the methane-air mixture is less satisfactory. The latter result is not surprising since the mechanism was not optimized for a pure methane fuel.
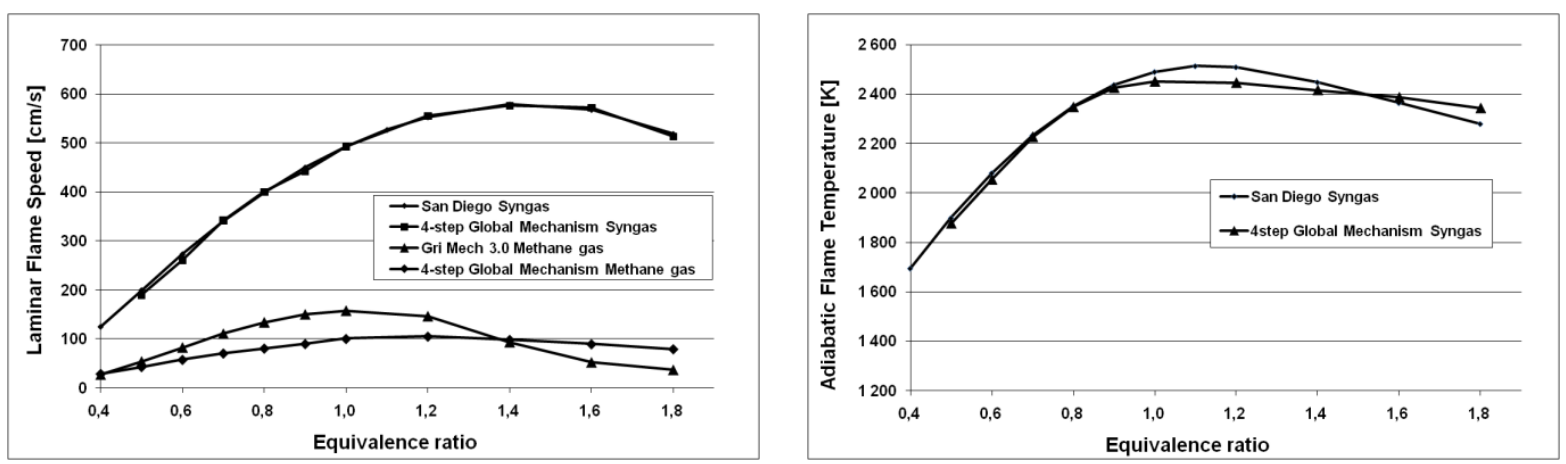

Figure 4: Gri Mech 3.0 and San Diego mechanism vs. optimized present scheme. Laminar flame speed for methane and syngas mixture (left) and syngas adiabatic flame temperature (right)

Figure 5 shows the equilibrium mole fractions of $\mathrm{CO}$ and $\mathrm{CO}_{2}$ vs. $\varphi$ for syngas. The agreement is very good for lean conditions. For rich conditions it is more difficult to match the emissions since the chemistry is more complex. This implies that one probably needs more reactions to be able to match the emissions sufficiently well at rich conditions. In spite of this, the results are in line with our expectations and fairly good. 

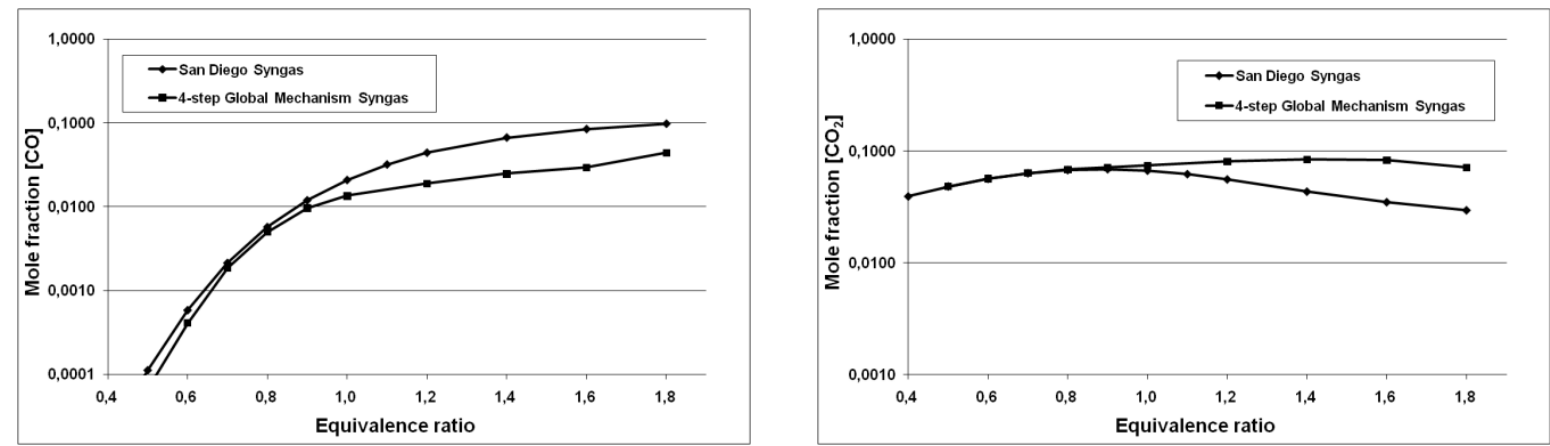

Figure 5: San Diego mechanism vs. optimized present scheme using syngas mixture. CO molar fraction (left up) and $\mathrm{CO}_{2}$ mass fraction (right up).

\section{Turbulence and Chemistry Interaction}

The combined model Finite Rate Chemistry/Eddy Dissipation Model (FRC/EDM), in Ansys CFX, was chosen for all CFD analyses. The FRC model computes the reaction rate $R_{k}$ from the following expression (equ. 2):

$$
R_{k}=\left(F_{K} \prod_{I=A, B, . .}^{N_{C}}[I]^{v_{k I}^{\prime}}-B_{K} \prod_{I=A, B, . . .}^{N_{C}}[I]^{v_{k I}^{\prime \prime}}\right)
$$

where $[\mathrm{I}]$ is the molar concentration of component $\mathrm{I}$ and $\mathrm{F}_{\mathrm{K}}$ and $\mathrm{B}_{\mathrm{K}}$ are the forward and backward rate constants respectively. $\mathrm{v}_{\mathrm{kI}}^{\prime}$ and $\mathrm{v}_{\mathrm{kI}}^{\prime \prime}$ represents the reaction order of component I component $\mathrm{I}$ in the reaction $\mathrm{k}$. The forward and backward rate constants assume Arrhenius temperature dependence as:

$$
F_{k}=A_{k} T^{\beta_{k}} e^{-\frac{E_{k}}{R T}} \quad \text { and } \quad B_{k}=\frac{F_{k}}{K_{C}}
$$

where $A_{k}$ is pre-exponential factor, $\beta_{k}$ is the temperature exponent, $E_{k}$ is the activation activation energy and $\mathrm{K}_{\mathrm{C}}$ the equilibrium constant. In the EDM model, the reaction rate of reaction $\mathrm{k}$ is computed as:

$$
R_{k}=A \frac{\varepsilon}{k} \min \left(\frac{[I]}{v_{k I}^{\prime}}\right)
$$

where $\mathrm{A}$ is a constant, $\frac{\varepsilon}{\mathrm{k}}$ is the turbulent mixing rate, $[\mathrm{I}]$ is the molar concentration of component $\mathrm{I}$ and $\mathrm{v}_{\mathrm{kI}}^{\prime}$ represent the reaction order of component $\mathrm{I}$ in the elementary reaction $\mathrm{k}$. The EDM model is based on the work of Magnussen and Hjertager [13]. The combined FRC-EDM model gives two different reaction rates for each reaction, one from the EDM model model and one from the FRC model. The minimum rate for each reaction is then chosen.

\section{CFD Modeling}

Time-averaged hybrid unsteady RANS/LES simulations were performed to predict flow and combustion dynamics. Simulations including combustion and non-reacting flow have been performed. The numerical prediction of the complex 3D swirling flow and the combustion process is computationally expensive. This implies that the boundary layer has not been resolved. The simulations were made on a $360^{\circ}$ model since there is no periodic condition in the model. 


\subsection{Boundary Conditions and Computational Mesh}

The CFD simulations contain all the complex 3D geometry from the experimental set-up, i.e. swirling vanes for MAIN and RPL systems, air and fuel cavities, cooling holes and ribs, etc. The specified mass flow is used as the boundary condition for the six inlets, see Figure 6. The total temperature for the fuel inlets are set to $300 \mathrm{~K}$ and the air inlets are preheated to $650 \mathrm{~K}$. The outlet boundary condition is set to atmospheric pressure, and all the walls are set to no-slip adiabatic walls. The equivalence ratio is set to 0.64 in the RPL and to 0.34 in the two remaining systems. The total equivalence ratio is approximately 0.35 in the burner. The reason for setting the total equivalence ratio to 0.35 in the CFD simulation and not to 0.31 as in the experiment, is due to the lean blow out limit. In the CFD, the lean blow out limit is obtained around equivalence ratio 0.34 .

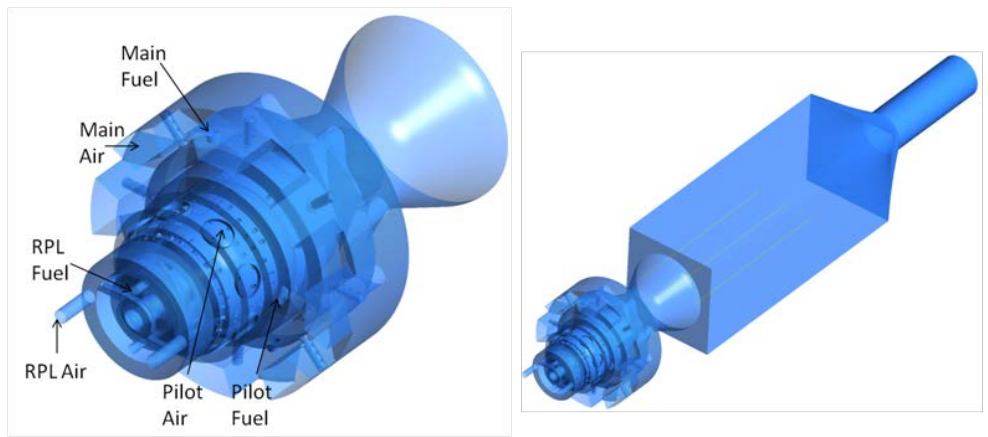

Figure 6: Configuration of the combustor inlets and liner

A mesh study has been performed in earlier work [7]. The chosen fine mesh is a multi-block structured mesh containing approximately 17 million hexahedral cells. Figure 7 shows the structured grid of the SIT flexi-fuel burner geometry. The ICEM CFD commercial software is used for the meshing.

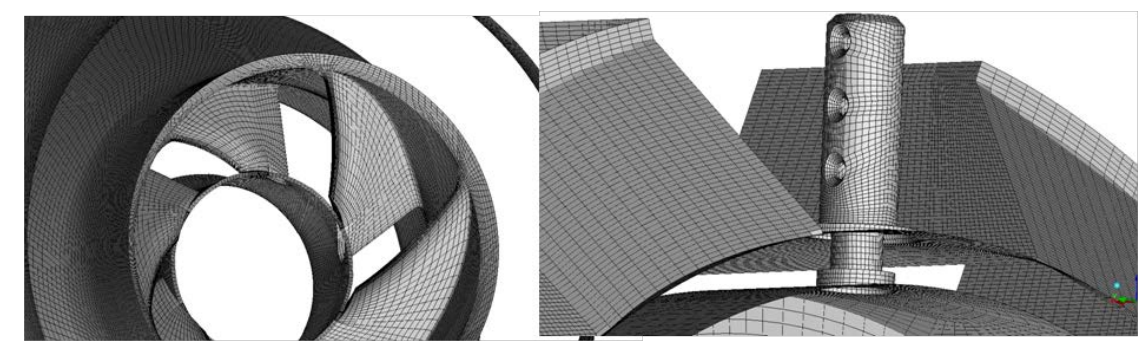

Figure 7: Mesh showing swirling vanes and fuel nozzles

\subsection{Numerical Method}

The Ansys CFX commercial software package was used to solve the governing equations. The Scale-Adaptive Simulation (SAS) turbulence model is used for the transient simulations. The SAS model is based on the introduction of the von Karman length scale into the turbulence scale equation. The information provided by the von Karman length scale allows the SAS model to adjust in order to resolve structures in a URANS simulation, which results in LES-like behavior in unsteady regions of the flow field. At the same time, the model provides standard RANS behavior in regions of stable flow [14]. The present global reaction mechanism was used in the transient simulation. 


\subsection{CFD Results}

\subsubsection{Axial velocities of non-reacting flow and reacting flow}

Figure 8 shows the mean axial velocity at the center axis using CFD compared to the measured data for non-reacting flow and reacting flow. The highest instantaneous and mean axial velocities occur in the beginning of the exhaust channel. For non-reacting flow the magnitude of the highest velocity is well captured, while the position is somewhat predicted upstream. The recirculation zone is slightly predicted larger than the experimental data. For reacting flow the magnitude of the highest velocity is well captured at centerline, while the position is not captured at all. At $y=15 \mathrm{~mm}$ off-center the agreement is good.
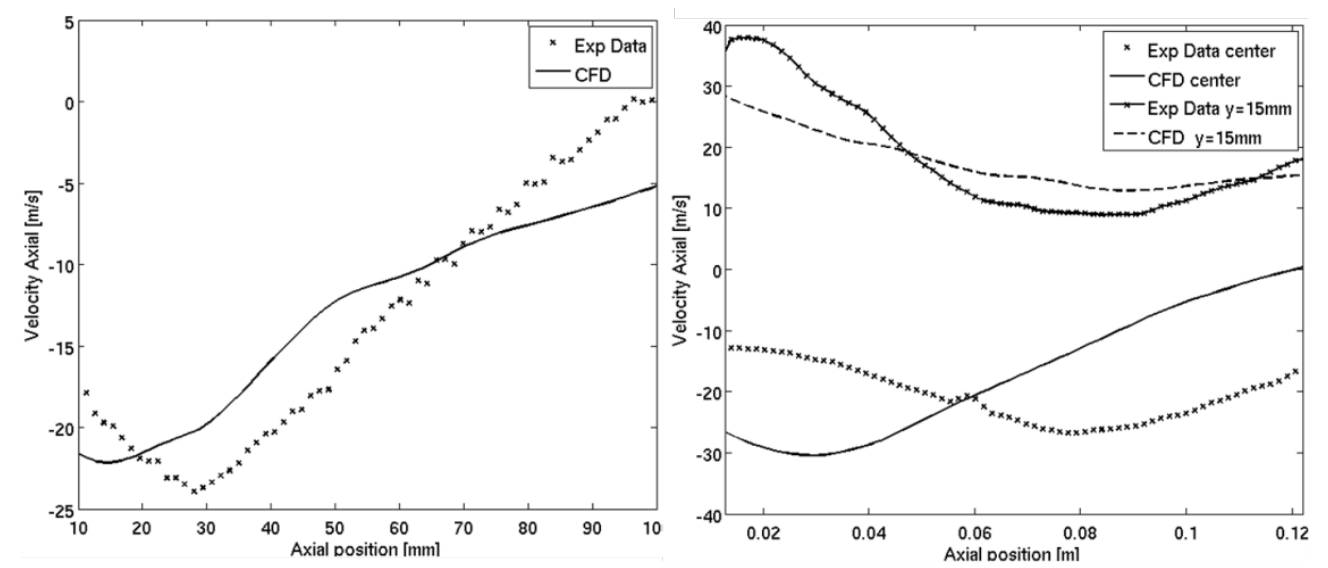

Figure 8: Axial velocities, CFD vs. Exp, non-reacting flow (left) and reacting flow (right)

\subsubsection{Temperature and emissions}

Figure 10 shows the averaged temperature at center line and a contour plot of the averaged temperature. The highest temperature is located in the beginning of the quarl part and is approximately $50 \mathrm{~K}$ below the adiabatic flame temperature at these conditions. In the end of the burner the temperature is $1585 \mathrm{~K}$. The adiabatic flame temperature for the syngas mixture, atmospheric pressure and an equivalence number of 0.35 is $1610 \mathrm{~K}$. This means that the present global reaction mechanism captures the temperature well.

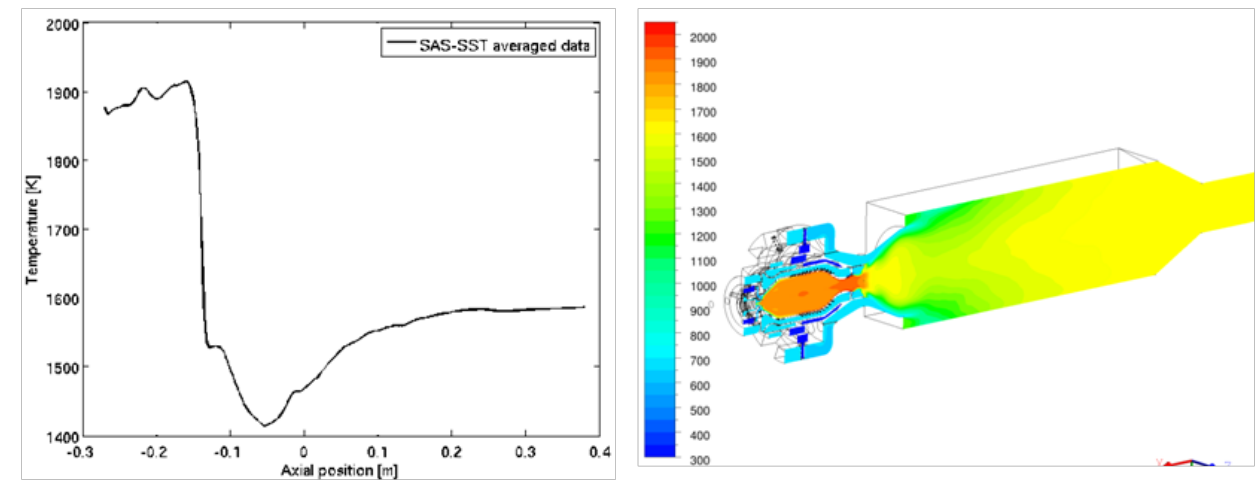

Figure 9: Transient averaged temperature $[\mathrm{K}]$ at centerline and a contour plot of the temperature $[\mathrm{K}]$

Figure 11 shows an averaged contour plot of the fuel composition. The $\mathrm{CO}$ and $\mathrm{H}_{2}$ components are consumed and produced. The $\mathrm{CO}$ fuel burns relatively slowly in comparison with the two other components. Since the amount of $\mathrm{H}_{2}$ is high in the fuel composition and is 
produced downstream in the liner, the amount of $\mathrm{H}_{2}$ is high downstream in the burner.

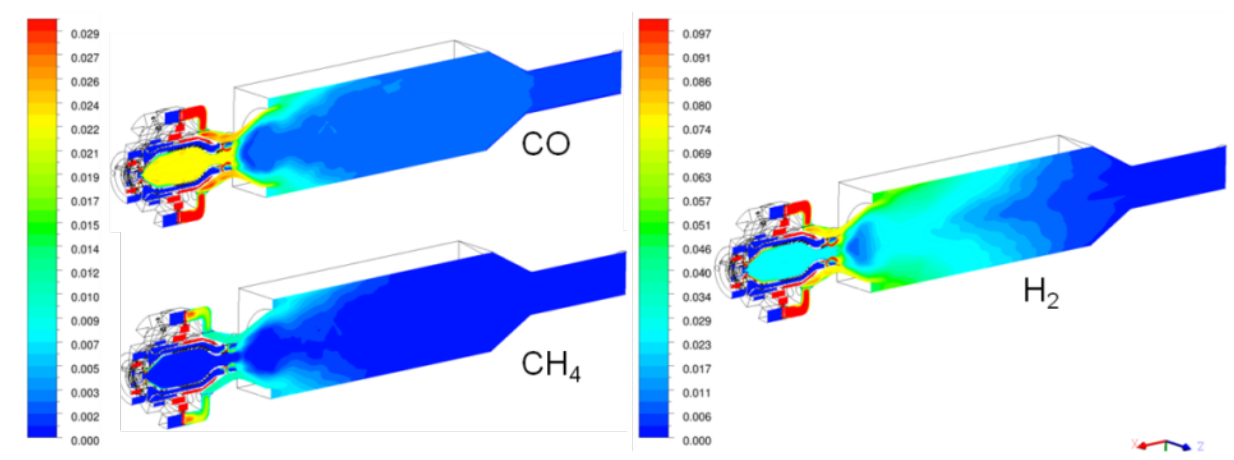

Figure 10: Transient averaged contour plots of the syngas fuel: $\mathrm{CH}_{4}, \mathrm{CO}$ and $\mathrm{H}_{2}$

\subsubsection{Flow field}

Figure 12 shows the iso-surface of the second invariant of the velocity gradient tensor, $\lambda_{2}$. The flow in the RPL part of the combustor is creating a vortex core extending from the inlet the quarl. This vortex core is strong and it is most likely to be caused by the swirling motion imposed by the swirler ducts upstream in the burner. Different core distributions are observed in the RPL part and the liner of the combustor. The liner consists of vortex rings breaking up rather quickly due to vortex-vortex interactions.
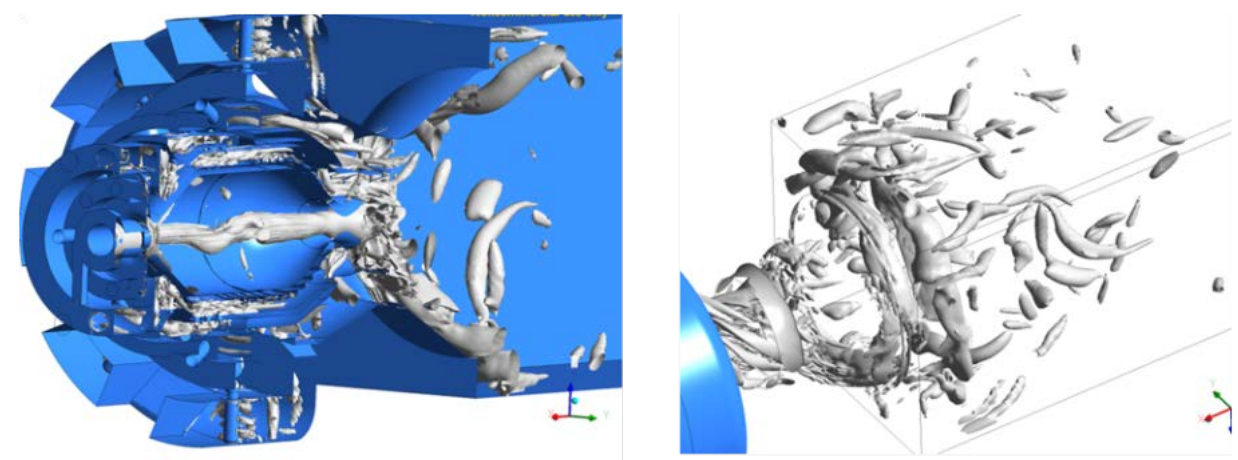

Figure 11: Isosurface of second invariant of the velocity gradient tensor

The mean flow field, length scales and proper orthogonal decomposition (POD) have been evaluated to specify the characteristics of the combustion flow. They are also used as comparison with the CFD result, to get the validity of the models used.

\subsubsection{Mean velocity field}

The mean velocity fields (Figure 13) show a strong recirculation zone in the central part of the burner. A stronger axial velocity can be found closer to the wall. There is a difference in location of the recirculation zone between the measured and the calculated flow fields (CFD). 


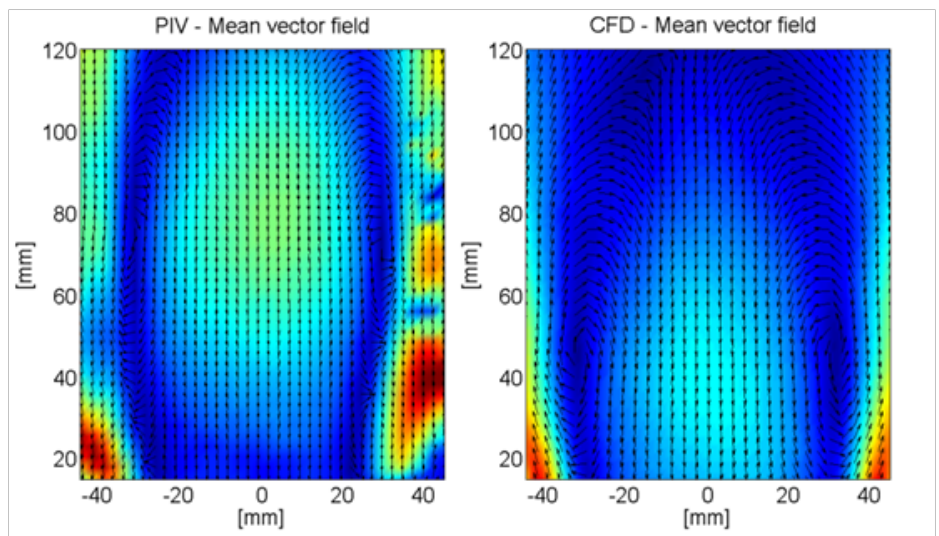

Figure 12: Mean velocity field. The transversal position $0 \mathrm{~mm}$ is at the centreline of the burner. The left image is the PIV results. To the right is the corresponding CFD result

\subsubsection{Planar Laser Induced Fluorescence Imaging - OH-pLIF}

A low laser fluoresce was used in these measurements to limit the likelihood of fluorescence saturation; as a consequence there was low signal to noise ratio in the images. The images have been processed to remove electronic noise, and are corrected for the vertical laser energy profile. Images shown have been additionally smoothed with an averaging filter (extent of 3 pixels). The images were cropped to eliminate reflections from the laser-liner interface. Shown in Figure 14 are two single shot images, the average of 500 images and CFD results. The two single shot images were chosen to illustrate the variability of the actively $\mathrm{OH}$ producing regions of the flame. Peak $\mathrm{OH}$ fluorescence can appear both at the upper edge of the measurement region and lower in the flame. Comparison of the PIV results (Figure 13) and the $\mathrm{OH}$ images (Figure 14) shows that the bulk of the $\mathrm{OH}$ emission is locate outside the stagnation zone, and follows the PIV trends at the upper and lower limits of the measurement region, i.e., contraction to the quarl diameter and rolling over in the upper reaches. The last image in Figure 14 shows the $\mathrm{H}_{2}$ mass fraction. The regions where the mostly of the $\mathrm{H}_{2}$ fuel is burning is similar to the regions where the highest $\mathrm{OH}$ emission are observed. Artifacts of the laser measurement can be seen as banding in the mean $\mathrm{OH}$ image, and should not confused with actual combustion phenomena.
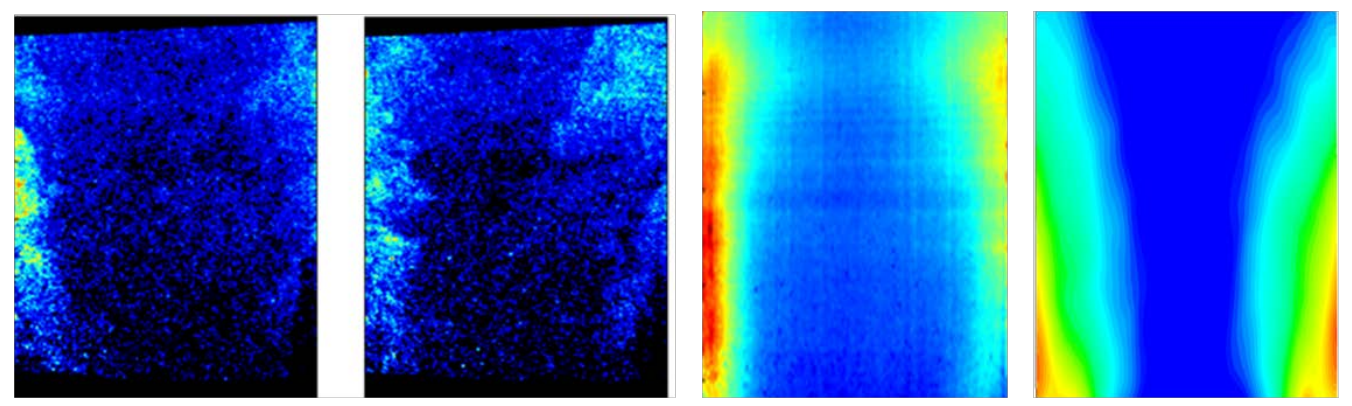

Figure 13: OH pLIF images (1-3) from SIT flexi-fuel burner at atmospheric pressure and image 4 from CFD. Two images to the left illustrate different locations of maximum $\mathrm{OH}$ emission. Third image is average $\mathrm{OH}$ pLIF from 500 images and the last image is averaged transient $\mathrm{H}_{2}$ from CFD

\subsubsection{Length and Time scales}

The integral scales and the Kolmogorov scales were calculated to specify which scales 
that are relevant to the measurements and the CFD. It also serves as a comparison of the measured flow and the CFD. In order to calculate the integral time scale, homogeneity is an important criteria [15-17]. Thus the point $25 \mathrm{~mm}$ at the centerline in the recirculation zone was used. The transverse velocity component ( $\mathrm{v}^{\prime}$ ) is homogeneous in the downstream direction from this point and the integral length scale is calculated from equation 5:

$$
l_{0} \equiv \int_{0}^{\infty} \rho(\Delta x) d \Delta x
$$

where

$$
\rho(\Delta x) \equiv \frac{\langle u(x) u(x+\Delta x)\rangle}{r m s(u(x)) r m s(u(x+\Delta x))}
$$

The transverse velocity component serves as the integral velocity. As the measurement data isn't time resolved the integral time scale is estimated as:

$$
t_{0} \equiv \frac{l_{0}}{v^{\prime}}
$$

The Reynolds number is based on the density and viscosity assuming complete combustion. The Kolmogorov scales are calculated according to Hinze [15]. It can be seen that the fluctuations is larger for the CFD calculations.

Table 3: Length scales

\begin{tabular}{|l|l|l|}
\hline Scales: & PIV measurements & CFD Calculations \\
\hline Integral length, $\mathrm{l}_{0}[\mathrm{~mm}]$ & 12 & 18 \\
\hline Integral time, $\mathrm{t}_{0}[\mathrm{~ms}]$ & 0.27 & 0.25 \\
\hline Integral velocity $\mathrm{v}^{\prime}[\mathrm{m} / \mathrm{s}]$ & 45 & 71 \\
\hline $\operatorname{Re}_{0}$ & 2200 & 5300 \\
\hline Kolmogorov length, $\eta[\mu \mathrm{m}]]$ & 37 & 29 \\
\hline Kolmogorov time, $\tau[\mu \mathrm{s}]$ & 5.7 & 3.4 \\
\hline
\end{tabular}

\subsubsection{Proper Orthogonal Decomposition (POD)}

To get an idea of how well the CFD data captures the dynamics of the flow the POD modes are calculated [18-21]. POD is a method of decomposing a large set of data in order to isolate important features. It is strongly related to linear curve fitting in space [22]. The modes are calculated by solving the eigen value problem stated below:

$$
[K][\varphi]=[\varphi][\lambda]
$$

where $[\mathrm{K}]$ is the covariance matrix of the velocities. The first 20 modes contain $99 \%$ of the dynamics in the system. Examination of the first modes illustrates that the CFD modeling is capturing main dynamics of the system. Shown here are modes 1 and 2 (Figure 15). It is easily seen that they are related. The main difference is that the structures are compressed towards the upstream section of the liner. This is the same phenomenon as for the mean flow (Figure 13). It should be mentioned that the POD from the PIV are based on 4000 images, which is enough to fully resolve the POD modes. The CFD POD modes are based on only 205 images, which is in the low range of resolving the modes. This is why mode 1 and mode 2 is switched in for the CFD POD. A test was performed by lowering the amount of images used for the PIV POD. When the amount of images was below 500 images, mode 1 and 2 was sometimes switched depending on which images that where included. 

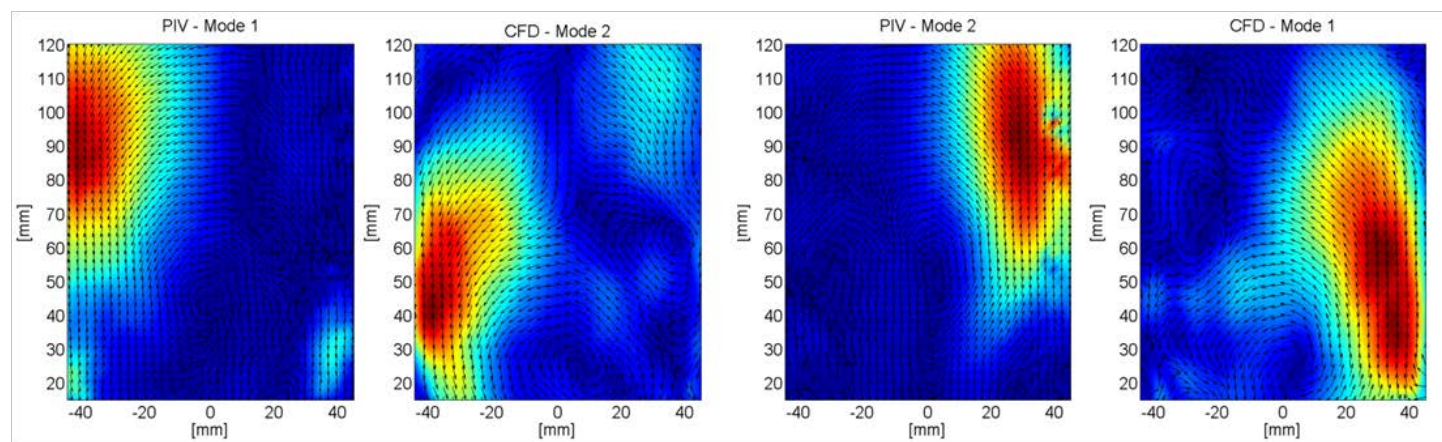

Figure 14: Left to right: PIV POD mode 1, CFD POD mode 2, PIV POD mode 2, CFD POD mode 1

\section{Conclusions}

A four-step scheme has been developed and evaluated in CFD. Comparisons of the four step reaction mechanism with the reference detailed reaction mechanism, San Diego, show that the adiabatic flame temperature, the laminar flame speed and the emissions are well predicted. The range of validity of the present global mechanism is equivalence ratios 0.4-1.8, atmospheric pressure and inlet temperatures corresponding to $295 \mathrm{~K}-650 \mathrm{~K}$. A test rig burner has been modeled with the optimized four-step global reaction mechanism using CFD tools. Comparisons were made with experimental data in the form of PIV data and OH-pLIF. The CFD results show that the velocity field, the temperature and the flame dynamics are well predicted compared to PIV and OH-pLIF data.

\section{Acknowledgements}

This research has been funded by the Swedish Energy Agency, Siemens Industrial Turbomachinery AB, Volvo Aero Corporation, and the Royal Institute of Technology through the Swedish research program TURBOPOWER, the support of which is gratefully acknowledged. Also, the computations were performed on C3SE computing resources. The help from Per Petersson and Jimmy Olofsson, Dantec Dynamics, with the high speed PIV system is appreciated.

\section{References}

1. O. A. Marzouk and E. D. Huckaby. A Comparative Study of Eight Finite-Rate Chemistry Kinetics for $\mathrm{CO} / \mathrm{H}_{2}$ Combustion. Engineering Applications of Computational Fluid Mechanics, Vol. 4, No. 3, pp 331-356, 2010.

2. N. Slavinskaya, M. Braun-Unkhoff and P.Frank. Reduced Reaction Mechanisms for Methane and Syngas Combustion in Gas Turbines. Journal of Engineering for Gas Turbines and Power, Vol.130, 2008.

3. Igor V. Novosselov and Philip C. Malte, 2008, "Development and application of an Eight-Step Global Mechanism for CFD and CRN Simulation of Lean-Premixed Combustors”, Journal of Engineering for Gas Turbines and Power, Vol.130.

4. P. Gokulakrishnan, S. Kwon, A.J. Hamer, M.S. Klassen and R.J. Roby, 2006, "Reduced Kinetic Mechanism for Reactive Flow Simulation of Syngas/Methane Combustion at Gas Turbine Conditions, ASME Turbo Power Expo, Power for land, Sea and Air, GT2006-90573. 
5. D. Marlow and T.S Norton, 1995, “A Reduced Mechanism for Low-Heating-Value Gas Combustion In a Perfectly Stirred Reactor”, American Flame Research Committee 1995 International Symposium

6. B. Franzelli, E. Riber, M. Sanjose and T. Poinsot. "Reduced Reaction Mechanisms for Methane and Syngas Combustion in Gas Turbines", Combustion and Flame, 175:1364-1373, 2010.

7. A. Abou-Taouk and L.E Eriksson. "Optimized Global Mechanisms For CFD Analysis Of Swirl-stabilized Syngas Burner for Gas Turbines”, 2011, ASME Turbo Power Expo, Power for land, Sea and Air, GT2011-45853, Vancouver, Canada.

8. A. Abou-Taouk, I. R. Sigfrid, R. Whiddon and Lars-Erik Eriksson, "CFD Investigation Of Swirl-Stabilized Flexi-Fuel Burner Using Methane-Air Mixture For Gas Turbines”, 2011, $20^{\text {th }}$ International Society for Airbreathing Engines, ISABE 2011-1122, Gothenburg, Sweden.

9. I. R. Sigfrid, R. Whiddon, R. Collin and J.Klingmann. Experimental Investigation of Lean Stability Limit of an Prototype Syngas Burner for Low Calorific Value Gases. ASME Turbo Power Expo, Power for land, Sea and Air, GT2011-45694, 2011.

10. I.R. Sigfrid, R. Whiddon, R. Collin and J.Klingmann, "Parametric Study of Emissions from Low Calorific Value Syngas Combustion, with Variation of Fuel Distribution, in a Prototype Three Sector Burner”, ASME Turbo Power Expo, Power for land, Sea and Air, GT2011-45689, 2011.

11. Smith, G. P., Golden, D. M., Frenklach, M., Moriarty, N. W., Eiteneer, B., Goldenberg, M., Bowman, C. T., Hanson, R. K., Song, S., Gardiner Jr, W. C., Lissianski, V. V., and Qui, Z., 1999, "Gri-Mech 3.0,"http://www. me. berkeley. edu/gri_mech

12. 2006, "San Diego Mechanism," http://maeweb.ucsd.edu/ combustion/cermech/

13. B. F. Magnussen and B. H. Hjertager. "On mathematical models of turbulent combustion with special emphasis on soot formation and combustion". 16th Symp. (Int'l.) on Combustion. The Combustion Institute, 1976.

14. Egorov, Y., and Menter, F., "Development and Application of SST-SAS Turbulence Model in the DESIDER Project", Second Symposium on Hybrid RANS-LES Methods, Corfu, Greece, 2007.

15. Hinze, J. O., 1975, Turbulence, Mcgraw-Hill Series in Mechanical Engineering, 99-0109193-4, McGraw-Hill, New York.

16. Pope, S. B., 2000, Turbulent Flows, Cambridge University Press, Cambridge.

17. Tennekes, H., and Lumley, J. L., 1972, A First Course in Turbulence, Cambridge, Mass.

18. Berkooz, G., Holmes, P., and Lumley, J. L., 1993, "The Proper Orthogonal Decomposition in the Analysis of Turbulent Flows," Annual Review of Fluid Mechanics, 25(1), pp. 539-575.

19. Chatterjee, A., 2000, "An Introduction to the Proper Orthogonal Decomposition," Current Science, 78(7), pp. 808-817.

20. Holmes, P., Lumley, J. L., and Berkooz, G., 1996, Turbulence, Coherent Structures, Dynamical Systems and Symmetry, Cambridge Monographs on Mechanics and Applied Mathematics,, Cambridge Univ. Press, Cambridge.

21. Sirovich, L., 1987, "Turbulence and the Dynamics of Coherent Structures," Quarterly of applied mathematics, 45(3), pp. 561-571.

22. Pearson, K., 1901, "Li. On Lines and Planes of Closest Fit to Systems of Points in Space," The London, Edinburgh, and Dublin Philosophical Magazine and Journal of Science, 2(11), pp. 559-572. 